\title{
Retraction notice for: Characteristics of liver fibrosis with different etiologies using a fully quantitative fibrosis assessment tool [Braz J Med Biol Res (2017) 50(6): e5234]
}

\author{
Q. $\mathrm{Wu}^{1,2}, \mathrm{X} . \mathrm{Zhao}^{1,2}$ and H. You ${ }^{1,2}$ \\ ${ }^{1}$ Beijing Key Laboratory of Translational Medicine in Liver Cirrhosis, Liver Research Center, Beijing Friendship Hospital, \\ Capital Medical University, Beijing, China \\ ${ }^{2}$ National Clinical Research Center for Digestive Diseases, Beijing, China
}

Retraction for: Braz J Med Biol Res | doi: 10.1590/1414-431x20175234 | PMID: 28538834

The authors would like to retract the article "Characteristics of liver fibrosis with different etiologies using a fully quantitative fibrosis assessment tool" that was published in volume 50 no. 6 (2017) (Epub May 18, 2017) in the Brazilian Journal of Medical and Biological Research <http://dx.doi.org/10.1590/1414-431x20175234 > PMID: 28538834.

The Corresponding author Hong You states that "I am the corresponding author of this article, but I have not reviewed any data or the manuscript; therefore I do not agree with the submission. Although the data is not false, it is inappropriate behavior. Therefore, after discussing with the other authors, we have decided to retract this article."

The email used by Hong You for this statement is different from the email used by the authors during the submission, evaluation, correspondence, and publishing processes with the Brazilian Journal. We regret the unprofessional behavior of the authors involved. 